\title{
10 JAHRE KOOPERATION E-MEDIEN ÖSTERREICH. BERICHT ÜBER DIE FESTVERANSTALTUNG AN DER VETERINÄRMEDIZI- NISCHEN UNIVERSITÄT WIEN (WIEN, 1. JULI 2015)
}

\section{von Helmut Hartmann}

Exakt am 1. Juli, dem offiziellen Gründungsdatum der Kooperation EMedien Österreich (KEMÖ), fand anlässlich des zehnjährigen Bestehens der KEMÖ im Festsaal der Veterinärmedizinischen Universität Wien eine gut besuchte ganztägige Festveranstaltung mit Beiträgen in- und ausländischer Fachleute zu verschiedenen Aspekten des Themenkreises Bibliothekskonsortien statt.

Als besonderes Zeichen der Wertschätzung der in und von der KEMÖ geleisteten Arbeit ließen es sich weder die Rektorin der Veterinärmedizinischen Universität Wien Dr. ${ }^{\text {in }}$ Sonja Hammerschmid noch MR Dr. Peter Seitz als Repräsentant des Bundesministeriums für Wissenschaft, Forschung und Wirtschaft nehmen, die Teilnehmerinnen und Teilnehmer zu begrüßen. Während Rektorin Hammerschmid die im Verhältnis zur Geschichte ihres Hauses, das 2015 sein 250-jähriges Bestehen feiert, kurze und dennoch so effiziente Zeit des Bestehens der KEMÖ thematisierte, stellte MR Seitz die imponierende Eigeninitiative der österreichischen Universitäts- und Fachhochschulbibliotheken, der Forschungseinrichtungen, der Nationalbibliothek sowie einiger Landesbibliotheken in den Mittelpunkt seiner Ansprache. Ohne jedwedes Zutun der ministeriellen Instanzen, ohne zusätzliche zentrale Mittel sei es ihnen gelungen ein nachhaltiges, höchst effizientes und sparsames Netzwerk ins Leben zu rufen, das den Bedürfnissen des modernen Bibliothekswesens voll und ganz entspreche und aus der Realität der österreichischen akademischen Bibliotheken nicht mehr wegzudenken sei.

HR Mag. Bruno Bauer, Leiter der Bibliothek der Medizinischen Universität Wien, würdigte als Vertreter des Forums Universitätsbibliotheken Österreichs (ubifo) in seiner Begrüßungsrede die besondere Rolle der KEMÖ als einigendes Bindeglied zunächst der österreichischen Universitätsbibliotheken, für die es als den einzelnen Universitäten eingegliederte Serviceeinrichtungen ex lege ja keine gemeinsame Planungsebene mehr gebe, darüber hinaus aber auch als ein gemeinsame Marktmacht begründendes 
Kommunikations- und Planungsnetzwerk aller wissenschaftlichen Bibliotheken Österreichs.

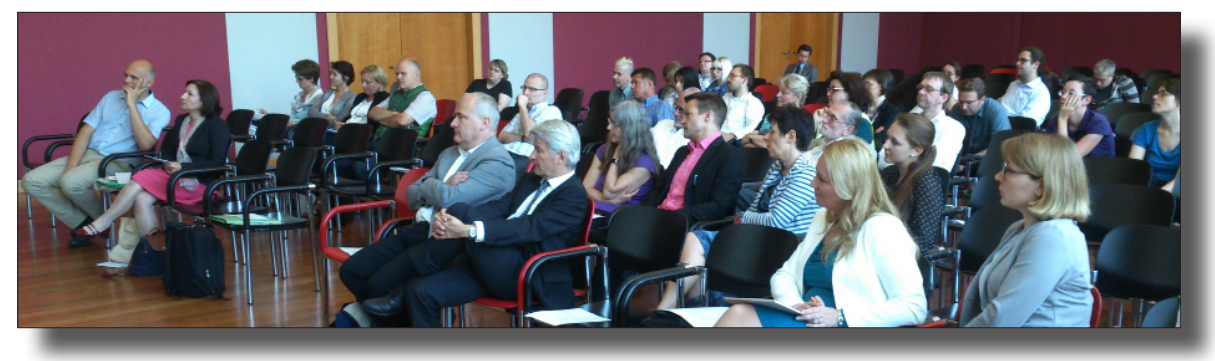

Abb. 1: Teilnehmerinnen und Teilnehmer der Festveranstaltung „10 Jahre KEMÖ“ an der Veterinärmedizinischen Universität Wien am 1. Juli 2015 (Foto: H. Hartmann)

Der darauf folgende erste Themenblock war dem „Blick über den Gartenzaun" gewidmet. Dr. in Hildegard Schäffler von der BSB München berichtete als Vorsitzende der GASCO in ihrem Beitrag „GASCO-Lizenzen, AllianzLizenzen, FID-Lizenzen: Eine konsortiale Standortbestimmung aus Deutschland“ über den gegenwärtigen Stand der Lizenzierungsebenen und -strukturen in Deutschland. Nach einem kurzen Überblick über die Organisation der GASCO und ihre Handlungsfelder arbeitete sie anschaulich die verschiedenen Merkmale von lokalen, gemeinschaftlich lizenzierten GASCO-/AIlianz-Lizenzen, community-basierten FID-Lizenzen und flächendeckenden DFG-geförderten Nationallizenzen heraus. Vor allem die in Österreich noch wenig bekannten FID-Lizenzen erregten großes Interesse. Dieses neue DFG-geförderte System der Fachinformationsdienste für die Wissenschaft (FID) löst das Sondersammelgebietssystem (SSG) ab und zielt u.a. auf die Realisierung einer e-only-policy bei der Versorgung mit Informationsressourcen des Spitzenbedarfs, also hochspezialisierten Ressourcen ab. Intendiert wird eine überregionale Versorgung einer jeweils spezifisch definierten Fachcommunity mit E-Ressourcen jenseits der klassischen "Campuslizenz" und damit jenseits existierender, institutionsbasierter Strukturen, wobei jedoch keine flächendeckende Versorgung angepeilt wird. Verhandelt werden diese Lizenzen im Rahmen eines Kompetenzzentrums (http://www.fid-lizenzen.de/) und/oder durch die FIDs selbst. Das Modell befindet sich noch in der experimentellen Phase und es ist noch nicht geklärt, wie es in die existierenden Lizenzierungsstrukturen eingefügt werden kann.

Darüber hinaus stellte Dr.in Schäffler eine Reihe flankierender lizenzierungsrelevanter Projekte vor: 
- das DFG-Projekt „Entwicklung eines Klassifikationsschemas (Banding) für die Informationsbeschaffung für wissenschaftliche Einrichtungen in Deutschland" (Zentralbibliothek des FZ Jülich, Bayerische Staatsbibliothek; abgeschlossen 2013) (http://www.allianzinitiative.de/fileadmin/user upload/redakteur/Banding-Studie-2014. pdf). Das Schema definiert fünf Einrichtungskategorien (Universitäten, Hochschulen ohne Promotionsrecht, Forschungseinrichtungen, Spezialbibliotheken, Staats- und Landesbibliotheken) mit jeweils sieben verschiedenen Größenstufen (Bands) auf der Basis von institutionen-adäquat definierten FTEs und der Anpassung der FTE-Bands für fachspezifische Produkte.

- das DFG-geförderte Projekt „Nationales Hosting elektronischer Ressourcen" (2014/15) (http://www.nathosting.de). Die Komponenten des erarbeiteten Konzepts sehen die Teilnahme eines nationalen Konsortiums bei Portico für die Inhalte größerer Verlage vor, weiters den Aufbau und Betrieb eines nationalen Private LOCKSS Network (PLN) mit wenigen verteilten Knoten mit Schwerpunkt auf „longtail“-Verlagen, den Aufbau und Betrieb einer rechnergestützten Rechteverwaltung durch das PLN sowie die Einrichtung einer Hosting-Agentur mit Koordinations- und Verhandlungsaufgaben.

- das DFG-Projekt (federführend hbz) zur Machbarkeitsanalyse und Konzeptionsentwicklung eines bundesweit verfügbaren, modular aufgebauten ERMS.

- die Musterlizenz für Lizenzverträge, die im Kontext der Allianz-Lizenzen entstanden ist (http://www.allianzinitiative.de/fileadmin/ user upload/redakteur/Musterlizenzvertrag 2013.pdf).

- den Statistikserver (Gauß'chen), der - entwickelt als DFG-Projekt an den UBs Frankfurt/M. und Freiburg/Br. - das automatische Einsammeln von COUNTER-Reports und die Bereitstellung insbesondere für National- und Allianz-Lizenzen sowie Konsortialstellen, Auswertungsmöglichkeiten ermöglicht und dessen Rollout für 2015 geplant ist.

Mag. ${ }^{a}$ Pascalia Boutsiouci, Leiterin der Geschäftsstelle des Konsortiums der Schweizer Hochschulbibliotheken, gab ihrem Bericht über den aktuellen Stand des Schweizer Konsortiums den Titel „Die Zeiten ändern sich und wir mit ihnen". Nach einem Rückblick auf die Anfänge im Jahr 2005 schilderte sie die gegenwärtige Lage anhand einiger Kennzahlen: 7 Mitarbeiterinnen und Mitarbeiter sind im Ausmaß von 4 FTEs in der Geschäftsstelle tätig. Sie betreuen rund 150 Produkte von 55 Verlagen in 75 Verträgen. Die Ressourcen 
sind zu $73 \%$ E-Zeitschriften, zu $23 \%$ Datenbanken und zu 4\% E-Bücher. Die 56 teilnehmenden Bibliotheken gehören zu Kantonalen Universitäten, Fachhochschulen, Pädagogischen Hochschulen, der ETH Zürich und anderen Einrichtungen. Zu den Kernaufgaben des Konsortiums zählen alle mit der Lizenzierung und der Administration von Ressourcen zusammenhängenden Dienstleistungen. Die Kommunikation mit den Bibliotheken wird über eine eigens entwickelte Multifunktionsplattform mit dem Namen "Sharepoint" abgewickelt. Sie dient auch der Information der Mitglieder und der Organisation und Dokumentation aller Arbeitsabläufe. Aktuelle Herausforderungen sind alle mit der Abbildung von Text- und Datamining zusammenhängenden Fragen und deren Abbildung in Verlagsverträgen, weiters die Integration von Open Access in die Verträge mit dem Ziel, Double Dipping zu verhindern und Verlags-PDFs auf Repositorien speichern zu dürfen. Ein besonderes Augenmerk gilt dem Problem der Vertraulichkeitsklauseln in den Verträgen, die in Widerspruch zum Bundesgesetz über das Öffentlichkeitsprinzip der Verwaltung stehen und in jüngster Zeit zum Gegenstand einer Klage wurden. Besonderes Interesse fanden die Ausführungen über das derzeit laufende Projekt der Etablierung von Nationallizenzen, die im Rahmen eines aufwendig geplanten Prozessmanagements im Laufe des kommenden Jahres zur Verfügung gestellt werden sollen. Abschließend berichtete die Referentin über eine bevorstehende Änderung der Organisationsform des Konsortiums: Während es seit seiner Gründung als Projekt an der ETH Zürich angesiedelt ist, soll nun eine Evaluation einer möglichen Abkoppelung von dieser Einrichtung erfolgen. Damit der Betrieb nach dem Jahr 2016 gesichert ist, muss rechtzeitig eine Rechtsform gefunden werden, die auch in Zukunft Nachhaltigkeit garantiert.

Den Block nach der Mittagspause, der sich unter dem Motto „Konsortien - immer noch ein Gewinn" mit der Beurteilung der in der KEMÖ geleisteten Arbeit aus dem Blickwinkel seiner Mitglieder befasste, eröffnete der langjährige Leiter und Mitbegründer der KEMÖ Helmut Hartmann mit einem als Impulsreferat gedachten Abriss der Entwicklung des Konsortiums unter dem Titel „10 Jahre KEMÖ in 10 Minuten“. Darauf folgte eine von der Stellvertretenden Leiterin der Geschäftsstelle Mag. ${ }^{a}$ Ute Sondergeld moderierte Podiumsdiskussion „Konsortien und die Struktur der KEMÖ aus der Sicht der Mitglieder". Dr.in Eveline Pipp von der Universitäts- und Landesbibliothek Tirol als Vertreterin der Universaluniversitäten, Dipl.-Bibl. (FH) David Frank von der Veterinärmedizinischen Universität als Vertreter der Fachuniversitäten, Mag. ${ }^{a}$ Andrea Zemanek von der FH Joanneum als Vertreterin der Fachhochschulen und Mag. a Elisabet Torggler vom Institut für Höhere Studien in Wien als Vertreterin der Forschungseinrichtungen diskutierten 
unter reger Beteiligung des Publikums die jeweils für ihren Einrichtungstyp spezifischen Anforderungen an die KEMÖ. Trotz mancher Unterschiede in der Priorisierung der verschiedenen Aufgaben stimmten alle vier Diskutantinnen und Diskutanten darin überein, dass die wesentlichste und unverzichtbare Leistung der KEMÖ die Servisierung der teilnehmenden Bibliotheken mit dem umfassenden Knowhow im Bereich der Vertragsverhandlungen und der Lizenzerwerbung sei.

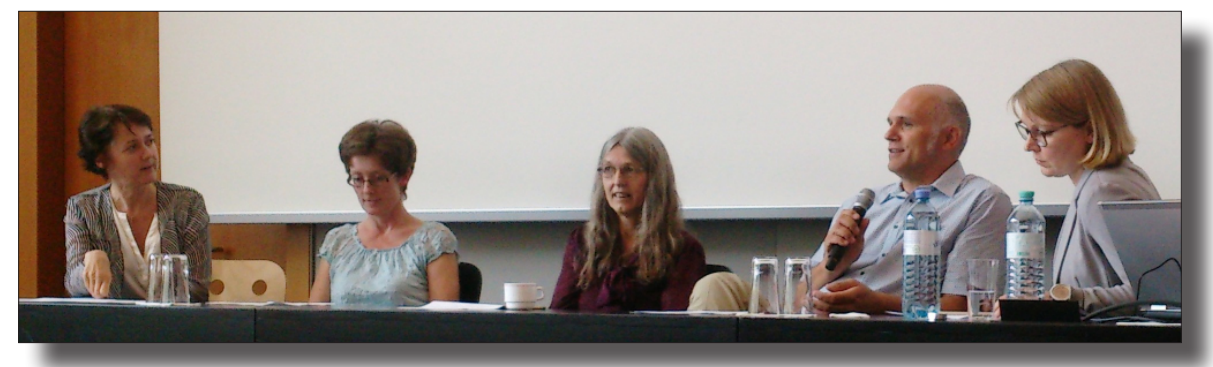

Abb. 2: Podiumsdiskussion im Rahmen der Festveranstaltung „10 Jahre KEMÖ“ an der Veterinärmedizinischen Universität Wien am 1. Juli 2015: Andrea Zemanek, Elisabet Torggler, Eveline Pipp, David Frank, Ute Sondergeld (v.l.n.r.) (Foto: H. Hartmann)

Der letzte Themenblock des Tages „Die nächsten zehn Jahre" wagte einen Blick in die Zukunft. Eröffnet wurde er mit einem Bericht von HR Mag. ${ }^{a}$ Brigitte Kromp von der UB Wien über „Konsortialverträge mit Open Access-Komponenten“. Die Referentin ging darin auf ihre in diesem jüngsten Bereich der Arbeit mit Online-Ressourcen gesammelten Erfahrungen ein. Sie spannte den Bogen von dem von ihr und Mag. ${ }^{a}$ Kerstin Stieg betreuten österreichischen Anteil am internationalen SCOAP3-Projekt über den international Aufsehen erregenden und unter Mitwirkung des Wissenschaftsfonds (FWF) zustande gekommenen Pionier-Vertrag mit dem Institute of Physics, der erstmals eine (partielle) Rückvergütung (Offsetting) der Article Processing Charges bei der Berechnung der Subskriptionsgebühren festschrieb und damit das doppelte Bezahlen an Verlage (Double Dipping) zu vermeiden helfen sollte, bis hin zum jüngsten Pilot Projekt Springer Compact, innerhalb dessen in den Jahren 2014-2016 34 Mitgliedseinrichtungen Zugriff auf über 2.000 Springer-Zeitschriften erhalten und ihren affilierten Wissenschaftlerinnen und Wissenschaftler über 1.600 Springer HybridZeitschriften für unkompliziertes Open Access-Publizieren zur Verfügung stellen können. Auch bei diesem Projekt spielt der FWF eine wichtige Rolle.

HR Mag. Bruno Bauer betätigte sich in seinem pointiert betitelten Beitrag „Konsortien - wie lange noch?" als advocatus diaboli, der selbstverständlich 
die Sinnhaftigkeit konsortialer Erwerbungsprozesse nicht ernsthaft in Frage stellte. Ausgehend von der Gründung der KEMÖ im Jahr 2005, ein Jahr nach dem Inkrafttreten des Universitätsgesetzes 2002, skizzierte er deren Erfolgsgeschichte. Obwohl weder für die Infrastruktur der Geschäftsstelle noch für die Lizenzierung von Konsortien zentrale Mitteln zur Verfügung standen, wurde die KEMÖ auf Initiative der Universitätsbibliotheken der öffentlichen Universitäten gegründet, beseelt vom Geist, dass die gemeinsame Lizenzierung von elektronischen Ressourcen für alle beteiligten Kooperationspartner Vorteile - inhaltlich wie finanziell - bringen werde. Nach der Öffnung der KEMÖ auch für andere Institutionen wuchs deren Mitgliederzahl sehr bald auf mehr als 50 Institutionen an. Dies ist insofern bemerkenswert, weil sämtliche Kosten für die Geschäftsstelle zu hundert Prozent von den Kooperationspartnern aufgebracht werden müssen. Weil die KEMÖ auf Dreijahresverträgen ohne automatische Verlängerungsmöglichkeit basiert, steht im Dreijahresrhythmus, jeweils zur Erstellung eines „KEMÖ-Folgevertrages“, eine Reflexionsphase an. 2015 erfolgte der Start der vierten Dreijahresperiode der KEMÖ. Die Personalausstattung steigerte sich in den zehn vergangenen Jahren von ursprünglich 0,6 FTE über 2 FTE auf mittlerweile 3 FTE. Parallel dazu hat sich auch das Aufgabenspektrum der KEMÖ deutlich erweitert. Weil Big Deals immer kritischer wahrgenommen werden, wurde im aktuellen Kooperationsvertrag als zusätzliche, zukunftsweisende Aufgabe „die gemeinsame Mitwirkung an nationalen und internationalen Open Access Initiativen" aufgenommen.

Dipl.Inf. Patrick Danowski und Mag. Márton Villányi vom IST Austria riskierten abschließend einen „Blick in die Glaskugel - KEMÖ 2025“. In suggestiven Bildcollagen warnten sie davor, die Arbeit der KEMÖ auf das bisherige Kerngeschäft der Ressourcen-Lizenzierung zu beschränken. Ohne langfristige Strategie und Erschließung neuer Geschäftstätigkeiten drohe sie ihre Daseinsberechtigung zu verlieren. Vor allem im Zusammenhang mit den immer wichtiger werdenden Open Access-Initiativen sei eine Erweiterung der KEMÖ in diese Richtung erforderlich, die sich auch in einer Namensänderung niederschlagen könnte: KEMOA. Als Drehscheibe für alle Aktivitäten der im Bereich Open Access tätigen Player FWF, OANA und Ministerium könne sie die Ausweitung des Open Access-Modells durch Koordination, Administration, Beratung, Evaluierung und nationale wie internationale Vernetzung vorantreiben. Nationallizenzen, EU-Lizenzen mit Offsetting auf EU-Ebene(!), Article Processing Charges-Pakete seien ebenso anzudenken wie Engagement in Aus- und Weiterbildung des Nachwuchses und eine fundierte Rechtsberatung. Darüber hinaus sei die „virtuelle KEMÖ“, also die Online-Plattform der KEMÖ, in weit höherem 
Ausmaß als bisher zu einer Kommunikations-, Informations- und Dokumentationsplattform auszubauen, die auch als anerkannte Schnittstelle zwischen Bibliotheken und Anbietern funktioniere.

In ihrem Schlusswort stellte sich die neue Leiterin der Geschäftsstelle Mag. ${ }^{a}$ Snježana Ćirković kurz vor und gab ihrer Freude Ausdruck, in Zukunft für die KEMÖ tätig sein zu dürfen. Als ihr besonderes Anliegen nannte sie einerseits die Intensivierung der Zusammenarbeit im Hinblick auf bestehende Mitglieder und Verträge, andererseits aber auch die Weiterentwicklung durch Öffnung für weitere Interessenten und Produkte und Aufgaben. Nach einem Wort des Dankes an allen Referentinnen und Referenten sowie den Teilnehmerinnen und Teilnehmern für ihr Kommen und Mitwirken und an die Leitung der Veterinärmedizinischen Universität für die Ausrichtung der Tagung ermunterte sie alle Festgäste, in das idyllisch an der Alten Donau gelegene Strandgasthaus „Birner“ zu übersiedeln und dort den Tag in angeregten Gesprächen ausklingen zu lassen.

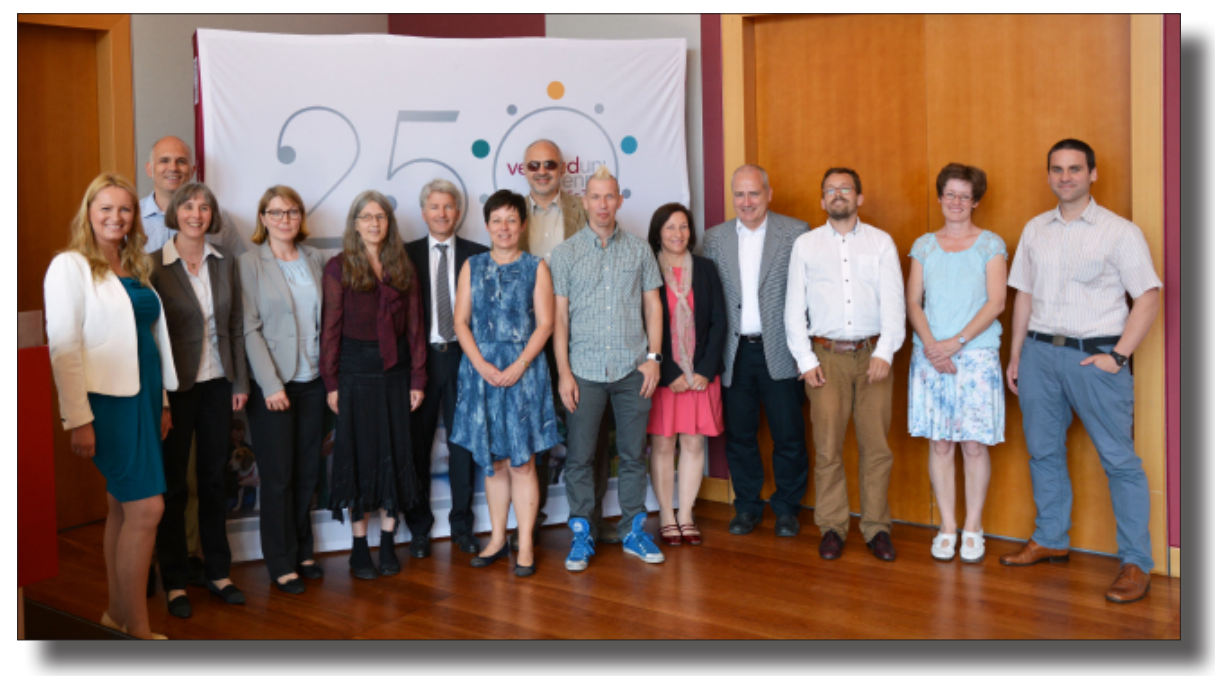

Abb. 3: Referentinnen und Referenten der Festveranstaltung „10 Jahre KEMÖ“ sowie das Team der Geschäftsstelle im Festsaal der Veterinärmedizinischen Universität Wien am 1. Juli 2015: Snježana Ćirković, David Frank, Hildegard Schäffler, Ute Sondergeld, Eveline Pipp, Peter Seitz, Brigitte Kromp, Helmut Hartmann, Patrick Danowski, Pascalia Boutsiouci, Bruno Bauer, Márton Villányi, Elisabet Torggler, Christof Köstl. [Andrea Zemanek und Rektorin Sonja Hammerschmid waren beim Fototermin leider nicht anwesend.] (Foto: G. Zippenfenig)

Helmut Hartmann E-Mail: helmut.hartmann@obvsg.at 